\title{
Physician Payment Disparities and Access to Services-a Look Across Specialties
}

\author{
John D. Goodson, $M D^{1,2}$, Sara Shahbazi, $P h D^{7}$, and Zirui Song, $M D, P h D^{1,2}$ \\ 'Division of General Internal Medicine, Department of Medicine, Massachusetts General Hospital, Boston, MA, USA; ${ }^{2}$ Harvard Medical School, \\ Boston, MA, USA.
}

J Gen Intern Med 34(11):2649-51

DOI: $10.1007 / \mathrm{s} 11606-019-05133-0$

(C) Society of General Internal Medicine 2019

$\mathrm{M}$ uch has been written about the shortage of primary care physicians. ${ }^{1}$ However, the supply and distribution of a whole range of specialties needed to care for an aging population raise similar concerns. Declining interest among trainees for certain specialties render potential disparities in access as an underappreciated issue in US health policy. Consider the following simple observation. Figure 1 shows the percent of counties in the USA that lack a single physician serving Medicare across a number of specialties, as well as the population in those counties. Relative to highly paid specialties, lower paid specialties are more likely to be absent in a county. While an outlier, the young but critically important specialty of addiction medicine, given the opioid epidemic, is currently absent in the vast majority of counties.

Among the many possible explanations, the lower reimbursements to certain specialties play a considerable role in career choice. The annual income for a radiologist or ophthalmologist, for example, is 1.7 and 1.6 times that of an infectious disease specialist. ${ }^{1}$ Medical students and residents are likely well aware of the economic consequences of their post graduate training choices. Although clinical preference and debt are known factors in career choice, the career income differentials that play out from top to bottom levels of compensation over 30 years of professional practice can be up to $\$ 5.1$ million using current reported incomes. ${ }^{1,2}$ Strategies to narrow gaps in access should consider the role of payment policy in workforce distribution.

Physician payment disparities have numerous root causes. Pricing for services for both public and private insurers, based on the premise of "relativity," was established by Congress in 1989. Over the last quarter century, the Resource-Based Relative Value Scale has established a rank order of intensity for payment purposes. The Centers for Medicare and Medicaid Services (CMS) definition of intensity includes "technical

Received March 7, 2019

Revised April 16, 2019

Accepted June 5, 2019

Published online August 5, 2019 skill, physical effort, mental effort and judgement and stress from patient risk." 3 The agency has relied heavily on the American Medical Association's Relative Value Scale Update Committee (RUC) to determine pricing. This group, largely comprised of representatives from procedurally oriented professional societies, has assigned relative value units (and thus prices) to thousands of existing and new services - most of which expand the repertoire of interventions and surgical procedures developed in recent years.

In contrast, no effort has been made over the last decade to update the definitions and valuations of the evaluation and management (E/M) services - the backbone and livelihood of our nation's cognitive specialists. With rare exception, historical valuations of $\mathrm{E} / \mathrm{M}$ services have not been revised to include new cognitive tools and non-procedural therapeutic options, including, but not limited to, motivational interviewing, shared decision-making, longitudinal follow-up care, and efforts to meet increasingly ambitious treatment goals for the most common chronic conditions (e.g., hypertension, diabetes, and high cholesterol). Moreover, the relative lack of representation of cognitive specialists on the RUC diminishes their role in defining and valuing (i.e., pricing) E/M services.

Failure to redefine and revalue E/M codes to reflect the complex cognitive work of contemporary clinical practice - coupled with the ability of physicians whose income is largely derived from procedures to improve efficiency and shorten intra-service times (thus fitting more procedures into the day) - has likely contributed to widening income disparities. These disparities have been accompanied by a geographic maldistribution of physician expertise as shown in Figure 1. For the Medicare population, $53 \%$ of US counties lacked an orthopedic surgeon serving Medicare, while $80 \%$ of counties lacked an infectious disease specialist serving Medicare. At the moment, $92 \%$ of counties lack an addiction medicine specialist serving Medicare. When population is considered, approximately 32 million Americans live in counties without an orthopedic surgeon serving Medicare, while 82 million Americans live in counties without an infectious disease specialist serving Medicare. About 232 million (also $92 \%$ of the population) Americans live in a county without an addiction medicine specialist serving Medicare. Again, higher paid specialties such as dermatology and ophthalmology are less absent among US counties. 


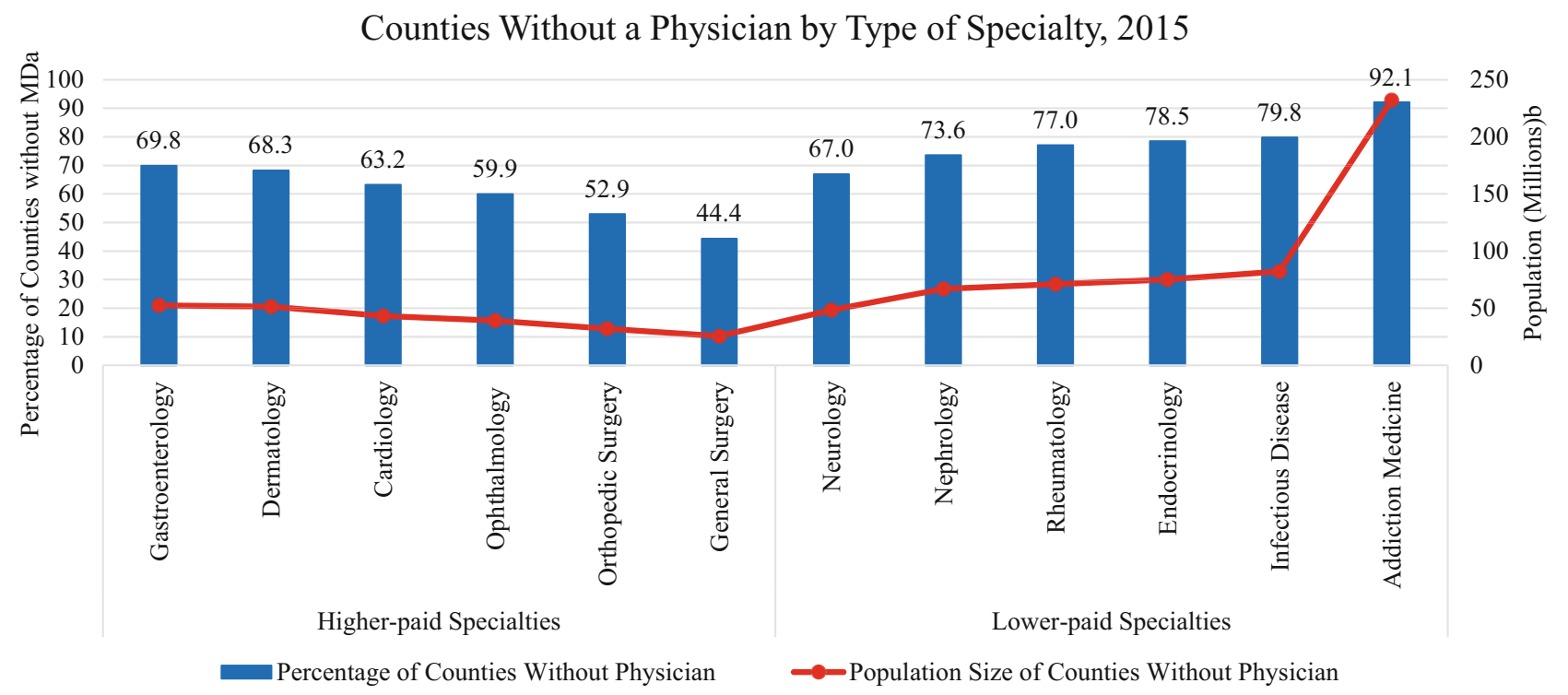

Figure 1 Counties without a specialist serving Medicare, by type of specialty. This graph maps the locations of all US physicians with any 2015 Medicare payment to the county of their billing. The percentage of the 3142 US counties that lacked a billing physician for each specialty is shown by the bar graph; the total population among those counties is shown by the red dots, connected by lines to facilitate comparisons of levels. Specialties were divided into lower paid and higher paid cohorts based on the Medscape Physician Compensation Report, 2015. Superscript letter " $a$ " indicates data from Medicare Provider Utilization and Payment Data: Physician and Other Supplier, 2015. Superscript letter "b" indicates data from US Census Bureau Population Division, Annual Estimates of the Resident Population: April 1, 2010 to July 1, 2016.

The consequences of this skewed distribution could be clinically and economically costly. For example, early infectious disease consultation in the care of Medicare patients is associated with $13 \%$ lower morality, as well as a lower readmission rate, length of stay, and costs. ${ }^{4}$ Amidst the growing opioid epidemic, early access to addiction treatment is increasingly important, but the current distribution of these specialists is limited.

CMS has recently begun to consider whether the existing outpatient $\mathrm{E} / \mathrm{M}$ codes are appropriately defined and valued relative to other physician services. Though the first proposal was for radical change - the collapsing of all payment to one level for new and one for established patients - the agency has since deferred final payment policy changes until 2021. This offers the opportunity to revisit the valuations of existing outpatient $\mathrm{E} / \mathrm{M}$ service codes and to understand how they could better serve the needs of the country through their distributional effects on trainees' decisions.

At the center of this opportunity is a reexamination of the status quo that cognitive specialists who serve medically complex and challenging patients face a pricing system that inadequately captures - and thus poorly values - cognitive services, relative to procedural services where the inputs into production are more clearly observable. The difficulty of observing effort is always a challenge in the valuation of cognitive work. However, to date, the fee schedule has offered no opportunity to recognize that the cognitive challenges posed by, for example, an elderly patient with multiple comorbidities might be more "intense" than some procedures. If payments for high-level cognitive care are not commiserate with the effort required, medical graduates will likely continue to forego the specialties lacked by most areas of the country, including infectious disease, endocrinology, rheumatology, and neurology, in addition to primary care.

While the aim of improving access to lower paid specialties is shared by many, debate around policy options is contentious. Currently, the pricing distortions that favor procedural services stem from inaccurate times that are used by the RUC to determine relative valuations and the inclusion of payment of services never delivered. Recent evidence shows that $\mathrm{E} / \mathrm{M}$ services built into the 10- or 90-day "global" payments for nearly all procedures are rarely provided $-4 \%$ in the former and $37 \%$ in the latter. ${ }^{5}$ Correcting these distortions is a potential area of agreement for physician payment policy.

Rebalancing physician payments to achieve a better workforce balance can be accomplished without major disruption if CMS asserts the importance of access. The agency has the authority and the resources to improve payments for $\mathrm{E} / \mathrm{M}$ services and increase the numbers of graduates choosing cognitive specialties that rely on the E/M codes. First, existing E/ $\mathrm{M}$ codes could be redefined and more appropriately valued based on valid and representative data from current-day practice and professional opinion. Second, payments for the undelivered care currently included within the global payments for procedures could cease. Third, valuations for procedures could be revised on a regular basis to include shorter intra-service times that reflect technological efficiencies and improvements. 
The opioid crisis and lack of addiction medicine specialists offer a cautionary tale. If a national infectious disease epidemic occurs, our infectious disease workforce may not be sufficient. Ultimately, a shift toward a more balanced workforce requires meaningful changes in how Medicare's $\$ 90$ billion of professional service payments are allocated.

Corresponding Author: John D. Goodson, MD; Division of General Internal Medicine, Department of Medicine Massachusetts General Hospital, Boston, MA, USA (e-mail: JGoodson1@MGH.Harvard.edu).

Funding Information This study is financially supported by a grant from the Office of the Director, National Institutes of Health (NIH Director's Early Independence Award, 1DP5OD024564, to Dr. Song).

\section{Compliance with Ethical Standards:}

Conflict of Interest: The authors declare that they do not have a conflict of interest.

\section{REFERENCES}

1. Kane L. Medscape Physician Compensation Report 2018. (https://www. medscape.com/slideshow/2018-compensation-overview-6009667\#4). Accessed 2019 Apr 4.

2. Leigh JP, Tancredi D, Jerant A, Romano PS, Kravitz RL. Lifetime Earnings for Physicians across Specialties. Med Care. 2012;50(12):1093101.

3. Centers for Medicare and Medicaid Services. Medicare program; revisions to payment policies under the Physician Fee Schedule and other revisions to Part B for CY 2019; Medicare Shared Savings Program requirements: Quality Payment Program; and Medicaid Promoting Interoperability Program. 2018. https://www.federalregister.gov/d/2018-14985. Accessed 2019 Apr 4.

4. Schmitt S, MacIntyre AT, Bleasdale SC, et al. Early Infectious Diseases Specialty Intervention Is Associated With Shorter Hospital Stays and Lower Readmission Rates: A Retrospective Cohort Study. Clin Infect Dis. 2019;68:239-46.

5. Kranz AM, Mulcahy A, Ruder T, Lovejoy S, Mehrotra A. Patterns of Postoperative Visits Among Medicare Fee-for-Service Beneficiaries. Ann Surg. 2018 .

Publisher's Note Springer Nature remains neutral with regard to jurisdictional claims in published maps and institutional affiliations. 